\title{
Investigating the functional interaction between semantic and episodic memory: Convergent behavioral and electrophysiological evidence for the role of familiarity
}

\author{
Andrea Greve, ${ }^{\mathrm{a}, *}$ Mark C.W. van Rossum, ${ }^{\mathrm{a}, 1}$ and David I. Donaldson ${ }^{\mathrm{b}, 2}$ \\ ${ }^{a}$ Neuroinformatics, University of Edinburgh, 5 Forrest Hill, Edinburgh, EH1 2QL, Scotland, UK \\ ${ }^{\mathrm{b}}$ Department of Psychology, University of Stirling, Stirling, FK9 4LA, Scotland, UK
}

Received 20 January 2006; revised 21 July 2006; accepted 24 July 2006

\begin{abstract}
Throughout our lives we acquire general knowledge about the world (semantic memory) while also retaining memories of specific events (episodic memory). Although these two forms of memory have been dissociated on the basis of neuropsychological data, it is clear that they typically function together during normal cognition. The goal of the present study was to investigate this interaction. One influence of semantic memory on episodic retrieval is 'Levels Of Processing'; recognition is enhanced when stimuli are processed in a semantically meaningful way. Studies examining this semantic processing advantage have largely concluded that semantic memory augments episodic retrieval primarily by enhancing recollection. The present study provides strong evidence for an alternative relationship between semantic and episodic memory. We employed a manipulation of the semantic coherence of to-be-remembered information (semantically related vs. unrelated word pairs) during an associative recognition memory test. Results revealed that associative recognition is significantly enhanced for semantically coherent material, and behavioral estimates (using the process dissociation procedure) demonstrated concomitant changes in the contribution of familiarity to retrieval. Neuroimaging data (event-related potentials recorded at test) also revealed a significant increase in familiarity based retrieval. The electrophysiological correlate of familiarity (the mid-frontal ERP old/ new effect) was larger for semantically related compared to unrelated word pairs, but no difference was present in the electrophysiological correlate of recollection (the left parietal old/new effect). We conclude that semantic memory and episodic memory do indeed interact in normal functioning, and not only by modulating recollection, but also by enhancing familiarity.
\end{abstract}

(C) 2006 Elsevier Inc. All rights reserved.

Keywords: Episodic memory; Semantic memory; Recollection; Familiarity; ERPs; PDP

\footnotetext{
* Corresponding author. Fax: +44 1316506899.

E-mail addresses: A.Greve@sms.ed.ac.uk (A. Greve), mvanross@inf.ed.ac.uk (M. C. W. van Rossum),did1@stir.ac.uk (D.I. Donaldson).

${ }^{1}$ Fax: +44 1316506899.

${ }^{2}$ Fax: +44 1786467641 .

Available online on ScienceDirect (www.sciencedirect.com).
}

\section{Introduction}

Human declarative memory supports conscious retrieval of information from the past and is widely believed to consist of two distinct systems (Tulving, 1972; Schacter and Tulving, 1994; Vargha-Khadem et al., 1997, but see Squire and Knowlton, 1995). Episodic memory refers to retrieval of personally experienced events embedded in the context of their acquisition (e.g., remembering a specific party), whereas semantic memory is retrieval of knowledge about the world without reference to any specific event (e.g., information about what generally happens at parties). Episodic and semantic memory are well characterized functionally, with behavioral and neuropsychological evidence strongly suggesting that they are dissociable.

Evidence that distinct systems support declarative memory comes from patient data (Mayes and Montaldi, 2001). Intact semantic but impaired episodic memory occurs in children with early-onset amnesia (Vargha-Khadem et al., 1997), whereas the opposite pattern of impairment occurs in developmental amnesia (Temple and Richardson, 2004). Furthermore, amnesic patients with impaired episodic memory are associated with bilateral hippocampal damage (Vargha-Khadem et al., 1997), whereas semantic dementia patients with impaired semantic memory have temporal lobe atrophy (Graham et al., 2000; Simons et al., 2001). This neuropsychological evidence suggests that episodic memory and semantic memory are supported by functionally and anatomically distinct brain systems. The proposal for anatomically distinct systems underlying episodic and semantic memory remains contentious; however, the data clearly demonstrate a functional dissociation between episodic and semantic memory in patient populations. This dissociation does not, of course, exclude the possibility that episodic memory and semantic memory interact in healthy participants.

Here we investigate the interaction between episodic and semantic memory, employing a manipulation of the semantic content of to-be-remembered stimuli and examining the effect on episodic retrieval. It is well known that semantic memory has an impact on performance (e.g. expertise effects, for review see 
Gobet, 1998). Similarly, several well established neuropsychological tests (e.g., the California Verbal Learning Test, Delis et al., 1987) assess the tendency to utilize semantic information inherent in the material that is studied and tested within an episodic memory paradigm. What is less clear, however, is exactly how semantic memory interacts with episodic retrieval. Dual-process models of episodic memory describe retrieval as contingent upon familiarity and recollection (Yonelinas, 2002). Familiarity refers to a fast acting process that reflects a quantitative assessment of memory strength, while recollection is the retrieval of qualitative contextual information about a previous event. Thus, our question is whether semantic memory influences episodic retrieval in terms of familiarity or recollection.

Event-related potentials (ERPs) provide an ideal method to investigate the engagement of retrieval processes during episodic memory tests because distinct ERP old/new effects (contrasting hits and correct rejections) have been identified as indexes of familiarity and recollection (see Johnson, 1995; Rugg, 1995; Donaldson et al., 2002, for reviews). Familiarity is reflected by an early mid-frontal ERP old/new effect, elicited around 300-500 ms post-stimulus onset, with maximum amplitude over frontal scalp sites (Curran, 2000; Nessler et al., 2001; although see Tsivilis et al., 2001). While debate continues as to whether the mid-frontal ERP old/new effect reflects familiarity when non-verbal stimuli are employed (e.g., faces, see Yovel and Paller, 2004), the effect has been widely used as a reliable index of familiarity in experiments using verbal stimuli (e.g., in studies of the revelation effect, see Azmimian-Faridani and Wilding, 2004). Recollection is indexed by the left parietal old/new effect, elicited around 500-800 ms post-stimulus onset, with maximum amplitude over the left temporo-parietal region (Paller and Kutas, 1992; Wilding et al., 1995; Rugg et al., 1998; for reviews see: Allan et al., 1998; Curran, 1999). Although these two ERP old/new effects are well characterized, ERP research has not directly investigated the question of how semantic memory influences successful episodic retrieval.

Current behavioral findings, most notably from Levels of Processing (LOP) studies (Craik and Lockhart, 1972), suggest that manipulations of semantic memory influence episodic retrieval largely by modulating recollection. The LOP theory states that episodic memory retrieval is influenced by semantic memory processing at encoding, with enhanced recognition for deep, semantically meaningful encoding (e.g., semantic categorization) compared to shallow non-semantic encoding (e.g., letter discrimination). Evidence suggests that deep encoding leads to recognition based on familiarity and recollection, while shallow encoding leads to recognition based primarily on familiarity (Mulligan and Hirshman, 1995; Toth, 1996; for review see Yonelinas, 2002). Thus, LOP strongly suggests an interaction between episodic and semantic memory which influences recollection to a greater extent than familiarity.

Further evidence that it is mainly recollection that is susceptible to semantic and episodic interactions is provided by Deese-Roediger-McDermott (DRM) false memory studies (for reviews, see Roediger and Bergman, 1998; Schacter, 1999). The DRM paradigm presents participants with a list of words, all semantically associated with a particular concept. In a subsequent recognition memory test, false alarm rates for unstudied words are much higher for words that are semantically associated than unassociated with the target concept. More importantly, these false alarm rates contain higher levels of false recollection compared to familiarity (Roediger and McDermott, 1995; Norman and Schacter, 1997). Although behavioral evidence strongly suggests that recollection is the source of false memories during the DRM paradigm, a recent ERP study by Nessler et al. (2001) suggests a possible role for familiarity. Specifically, Nessler et al. found that differences between true and false memory are influenced by strategic processes engaged during encoding (such as processing conceptual features of the stimuli), and that this is reflected primarily in the ERP correlates of familiarity. False memory data are, therefore, somewhat difficult to reconcile, with the ERP findings apparently sitting at odds with behavioral findings.

While behavioral evidence from both LOP and DRM studies suggests that it is primarily recollection that mediates the interaction between episodic and semantic memory, other evidence suggests that familiarity may also play a role. For example, the Speed-Accuracy Trade-off (SAT) method has been used to test recognition for semantically related and unrelated word pairs, revealing a maximum false-alarm rate to semantically related lures at short retrieval deadlines (Dosher, 1984). Whereas retrieval based on recollection is relatively slow, familiarity is regarded as a fast acting process, suggesting that the false alarms to semantically related pairs were due to familiarity. This finding suggests, therefore, that familiarity, but not recollection, might be more affected by manipulations of semantic memory.

In general, previous studies do not provide a conclusive account as to which episodic retrieval process (familiarity or recollection) mediates the interaction between semantic and episodic memory. The present study is designed to directly investigate this question, examining the functional and neural correlates of successful episodic retrieval. To examine the interaction between episodic and semantic memory we manipulated the semantic coherence of to-be-remembered information, requiring the retrieval of categorically related vs. unrelated word pairs during an associative recognition test. We employed neuroimaging (event-related potentials) and behavioral (process dissociation procedure) measures to provide convergent evidence that semantic memory does indeed influence episodic retrieval. We predicted that episodic retrieval would be enhanced for semantically coherent word pairs, and based largely on LOP theory, that the increase in performance would primarily be associated with greater recollection.

\section{Materials and methods}

\section{Participants}

All participants were right-handed native English speakers, ranging from 18 to 35 years in age, with normal or corrected-to-normal vision and no known neurological problems. Informed consent was required, and payment provided at a rate of $£ 5$ per hour. Twenty-two participants performed the ERP study; seven were excluded due to either technical failure or EEG artifact, and the remaining 15 (6 female) had an average age of 24 years. An additional 12 participants ( 8 females, mean age 21) performed a follow-up behavioral study using the process dissociation procedure.

\section{Materials}

Stimuli consisted of the presentation of a category name followed by a word pair. Each word pair was classed as being 
either semantic (one or both words were category exemplars ${ }^{3}$ ) or non-semantic (both words were completely unrelated to the category) as shown in Fig. 1. Word pairs were constructed from 864 nouns, 4-6 letters in length, with a frequency between 10 and 30 occurrences per million (Kucera and Francis, 1967). A third (288) of the nouns were category exemplars (12 nouns for each of 24 categories), the remainder (576) were unrelated to the categories.

Stimuli were created for 6 blocks, each containing a single study list of 32 semantic and 32 non-semantic word pairs (randomly intermixed), matched with a corresponding test list of 24 semantic and 24 non-semantic word pairs. Test lists were created for each condition (semantic and non-semantic) in the following way: 8 pairs were re-presented at test in the same pairings as at study, providing 8 intact word pairs; 16 pairs shown at study were recombined to create 8 rearranged word pairs by taking one word from each of two study pairs (maintaining word position in each case) and discarding the other member of each study pair. To be clear, rearranged study word pairs A-B and C-D would be recombined to form $\mathrm{A}-\mathrm{D}$, discarding $\mathrm{B}$ and $\mathrm{C}$ (i.e., the first word and second words of a study pair were discarded equally often). The remaining 8 pairs were used to create 8 new word pairs, by combing the first member of each pair (always discarding the second member of the pair) with a previously unstudied word. The resulting semantic and non-semantic word pairs for each of the intact, impaired and new conditions were randomly intermixed within each test list.

\section{Procedures}

The experiment was implemented using E-Prime and a PST Serial Response Box. All stimuli were displayed in white, using upper case Courier New 18 point font, against a black background. Category names were displayed in central vision, and word pairs were displayed one above the other, slightly above and below central fixation. The category name associated with an individual word was always the same at study and test (thus when pairs were rearranged this was performed within each category). At the viewing distance of approximately $1 \mathrm{~m}$, the stimuli subtended a maximum visual angle of $3.7^{\circ}$ horizontally and $1.4^{\circ}$ vertically. The 6 study-test blocks were presented in a random order for each participant. An additional practice list was always shown first to familiarize participants with the procedure. Fig. 1 illustrates the design. The factors of relationship (semantic, non-semantic) and pairing (intact, impaired, new) were fully crossed within participants.

Each study trial started with a fixation cross $(+)$ displayed for $750 \mathrm{~ms}$ in the center of the screen, followed by a blank screen for $250 \mathrm{~ms}$. The category name was then presented for $1500 \mathrm{~ms}$ before being replaced by a word pair for $2000 \mathrm{~ms}$. Participants were instructed to indicate by button press (within $5750 \mathrm{~ms}$ of word pair onset) whether none, one or two of the words were related to the category name. The study task was intended to ensure that the

\footnotetext{
${ }^{3}$ We initially distinguished between high (50\%) and low (50\%) semantic word pairs (one or both words being related to the category name respectively). However, analysis of the behavioral results revealed no differences between performance for high and low semantic conditions, and there were insufficient trial numbers to create separate ERPs. The behavioral and ERP data are therefore collapsed across high and low semantic word pair conditions.
}

semantic meaning of the word pairs was encoded. Once a response was made, the screen went blank for $250 \mathrm{~ms}$, and the next trial began. Participants were then informed that their memory would be tested.

Each test trial started with an initial $750 \mathrm{~ms}$ fixation cross, followed by a $250 \mathrm{~ms}$ blank screen, a $1500 \mathrm{~ms}$ category name and then a word pair for $2000 \mathrm{~ms}$. Category names were re-presented at test, ensuring that the semantic coherence of the stimuli remained salient, and therefore augmenting the influence of the category on performance. Participants were instructed to indicate by button press (within $7750 \mathrm{~ms}$ of word pair onset) whether each word pair was a target (respond: old) or a non-target (respond: new). Targets were defined as intact word pairs; non-target as rearranged and new word pairs. Once a response was made, a short $(250 \mathrm{~ms})$ delay occurred, and participants were then prompted to rate their response confidence by button press on a five point scale ( 1 =guess, $2=$ unsure, $3=$ think so, $4=$ pretty sure, $5=$ certain) . Once a confidence response was made, the screen went blank for $250 \mathrm{~ms}$, and the next trial began. Participants were asked to respond as quickly and accurately as possible, and the mapping of buttons to old and new responses was counterbalanced across participants.

The initial ERP study was followed with a behavioral study based on the process dissociation procedure (Jacoby, 1991), which allows estimates of the contribution of familiarity and recollection to be calculated based on differences in performance in 'exclusion' and 'inclusion' tests. The ERP study outlined above is an exclusion test, whereby rearranged pairs had to be excluded as non-targets. The inclusion test is identical to the exclusion test with exception of the task instructions given to participants. In the inclusion version, an 'old' response is required for intact and rearranged word pairs (target trials) while a 'new' response is required only for new word pairs (non-target trials). By comparing performance on exclusion and inclusion tasks the process dissociation procedure (PDP) provides estimates of the contribution of recollection and familiarity. PDP estimates are based on the assumption that an 'old' response to recombined pairs is given in the inclusion task when either the pair was recollected $(R)$ or when the pair was familiar $(F)$ in the absence of recollection $(1-R)$, i.e. $P$ (old inclusion $)=R+(1-R) F$. In contrast, an 'old' response to recombined pairs in the exclusion task should only be evoked by familiarity $(F)$ in the absence of recollection $(1-R)$, i.e. $P$ (old exclusion $)=(1-R) F$. Recollection of the precise pairing in the exclusion task would lead to a correct rejection of the recombined pair. These processing differences for inclusion and exclusion responses allow the derivation of the following estimates for familiarity and recollection:

Recollection $=P($ old $\mid$ inclusion $)-P($ old $\mid$ exclusion $)$.

Familiarity $=P($ old $\mid$ exclusion $) /(1-R)$.

Inclusion and exclusion experiments are typically performed within participants, to avoid differences in response bias across tasks and to allow statistical analysis of the resulting estimates. We therefore carried out an additional behavioral experiment to obtain PDP estimate of familiarity and recollection using the same stimuli as in the ERP study. Only two changes were made from the original paradigm. First, subjects performed both inclusion and exclusion versions of the experiment, as outlined above; task order was counterbalanced across participants. Second, because two 

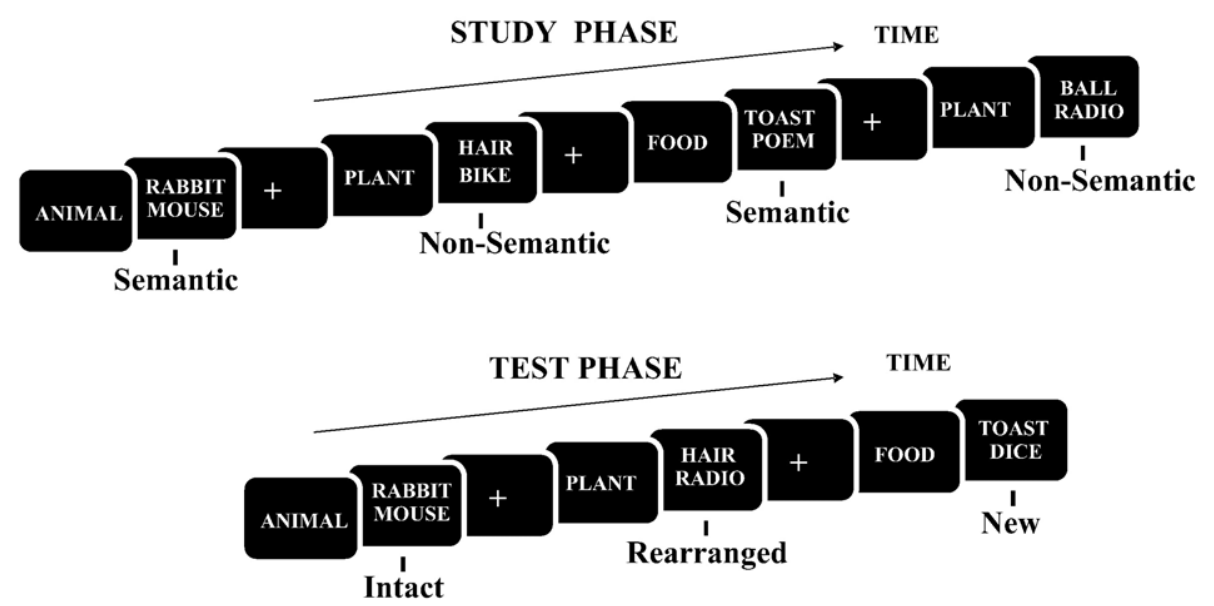

Fig. 1. The experimental design and materials. During the study phase each trial involved the presentation of a category name followed by a word pair. Either one or both of the words were semantically related to the category (semantic), or neither of the words contained a semantic relation to the category (non-semantic). During the test phase the same trial procedure was employed and three types of word pairs were presented: intact pairs, which were previously presented at study, rearranged pairs, which were constructed by recombining words from two study pairs and new pairs, which were constructed by combining a member of a studied pair with an unstudied word. Intact, rearranged and new word pairs were constructed separately for the semantic and non-semantic conditions.

tasks were being performed by each participant rather than one, the number of inclusion and exclusion trials was reduced by half (leaving the total number of trials the same).

\section{ERP recording and analysis}

Scalp EEG was recorded from 61 Silver/Silver-Chloride electrodes embedded in a cap based on an extended version of the international 10-20 system (Jasper, 1958): Fz, FCz, Cz, CPz, Pz, POz, Oz, FP1, FP2, AF7, AF8, AF3, AF4, F7, F8, F5, F6, F3, F4, F1, F2, FT7, FT8, FC5, FC6, FC3, FC4, FC1, FC2, T7, T8, C5, C6, C3, C4, C1, C2, TP7, TP8, CP5, CP6, CP3, CP4, CP1, CP2, P7, P8, P5, P6, P3, P4, P1, P2, PO7, PO8, PO5, PO6, PO3, $\mathrm{PO} 4, \mathrm{O} 1, \mathrm{O} 2$. Data were recorded and processed using Neuroscan 4.2 software. EEG was recorded using a left mastoid reference, but EEG was also recorded from the right mastoid, allowing rereferencing off-line to recreate linked mastoid recording. EOG electrodes were located above and below the left eye for monitoring eye blinks and on the outer canthi of both eyes to monitor lateral eye movements. Electrode impedances were adjusted below $5 \mathrm{k} \Omega$, and all data were recorded with a bandpass filter of $0.1-40 \mathrm{~Hz}$ and digitized (16 bit) at a rate of $8 \mathrm{~ms}$ per point. Eye-blink artifacts were removed using a regression procedure (Neuroscan Ocular Artifact Reduction), calculating an average blink from a minimum of 32 blinks for each participant, and removing the contribution of the blink from all other channels on a point-by-point basis.

Continuous EEG data were separated into $2000 \mathrm{~ms}$ epochs, beginning $200 \mathrm{~ms}$ before the onset of each word pair. Individual epochs were baseline corrected and smoothed over 5 points. Epochs were excluded (average 9\%, ranging from 4\% to 19\%) when eye movements were present (horizontal electrooculogram (EOG) effects greater than $100 \mu \mathrm{V}$ ), when any channel became saturated (exceeding $\pm 495 \mu \mathrm{V}$ ) or when baseline drift (absolute difference in amplitude between the first and last data point of each individual epoch) was greater than $75 \mu \mathrm{V}$ on any EEG channel. A minimum of 16 artifact-free trials were required from each participant in each condition to ensure an acceptable signal-to-noise ratio. To minimize
EEG artifacts, participants were asked to relax, minimize body, head and eye movements, and to fixate on the center of the screen.

As noted above, the experiment was designed to examine the ERP old/new effects associated with the retrieval of semantic and non-semantic word pairs, thus ERPs were formed for correct responses to intact and new word pairs. ERP analyses were designed to investigate the pattern of old/new effects in the semantic and non-semantic conditions, revealing any differences in the engagement of the generators of the left parietal and midfrontal ERP old/new effects. ERPs to rearranged pairs were not examined because there were insufficient artifact free trials in this condition. Based on previous findings and visual inspection of the waveforms, data were analyzed over 4 consecutive time windows (300-600 ms, 600-900 ms, 900-1200 ms and 1200-2000 ms). The first two time windows capture the ERP correlates of familiarity and recollection; the mid-frontal and left parietal old/new effects. As other late-onsetting old/new effects have been reported, the later time windows were also included in the analysis.

All statistical tests were conducted with a significance level of 0.05. Analysis of ERP data employed the Greenhouse-Geisser correction for non-sphericity, and corrected $d f$ and $p$ values are reported as appropriate. All topographical analyses were performed on difference scores (subtracting the ERPs for new word pairs from those for intact word pairs) and these effects were normalized prior to analysis using the Max-Min method (McCarthy and Wood, 1985).

\section{Results}

\section{Behavioral data}

Fig. 2 shows the probability of a correct response (bars) for intact, rearranged and new word pairs, along with associated reaction times (lines). The data are shown separately for semantic and non-semantic word pairs, demonstrating a clear improvement in recognition performance for semantically related compared to unrelated pairs. Importantly, analysis revealed that participants were able to discriminate between the different types of word pairs 
in both conditions (paired $t$-tests comparing hits (intact) vs. false alarms (rearranged), and hits (intact) vs. false alarms (new) were significant $(p<0.001)$ in all comparisons).

$\mathrm{Old} /$ new discrimination accuracy $[\mathrm{Pr}=\mathrm{Hit}-\mathrm{FA}]$ and response biases $[\mathrm{Br}=\mathrm{FA} /(1-\mathrm{Pr})]$ were computed for semantic and nonsemantic conditions, whereby Hit is the probability of 'old' response to an intact pair, and FA is the probability of an 'old' response to a new pair. Discrimination accuracy was significantly better for the semantic (0.48) than non-semantic (0.29) conditions $(t(14)=4.68$, $p<0.001)$. Similarly, response bias was significantly higher for the semantic $(0.56)$ than non-semantic $(0.41)$ conditions $(t(14)=3.77$, $p<0.05)$. We considered the possibility that the two high threshold model might not provide a suitable fit for the target/new discrimination and calculated the response bias based on detection theory. Nevertheless, the pattern of results remained the same as a more liberal response bias was found for the semantic condition. Whether this difference in performance for semantic and nonsemantic pairs is reflected in the ERP data will be discussed later.

The statistical analysis of the behavioral data was performed with an ANOVA including the factors of relationship (semantic, non-semantic) and pairing (intact, impaired, new). Results revealed a significant main effect of pairing $[F(2,28)=21.31, p<0.001]$, no main effect of relationship [ $p>0.05]$, but a significant interaction between relationship and pairing $[F(2,28)=33.82, p<0.001]$. As Fig. 2 shows, intact pairs were better remembered when semantically related $(t(14)=7.56, p<0.001)$. By contrast, rearranged pairs were more difficult to reject when semantically related $(t(14)=4.38, p=0.001)$, and new pairs were equally well rejected in the semantic and non-semantic conditions $(p>0.05)$.

Reaction time data in Fig. 2 (lines) reveal no clear influence of the manipulation of semantic relationship. An ANOVA with the factors of relationship (semantic, non-semantic) and pairing (intact, impaired, new) revealed no significant main effects or interactions [ $p>0.05$ in all cases]. Nonetheless, the behavioral results clearly demonstrate that semantically related word pairs were better remembered than unrelated pairs.

Confidence ratings for the semantic and non-semantic pairs were compared. The analysis tested whether the memory advantage for semantic vs. non-semantic intact pairs is carried solely by the 'high confidence old' ratings, which are expected to reflect recollection in addition to familiarity, or by 'middle range confidence old' (i.e., 2 to 4) ratings, which indicate the

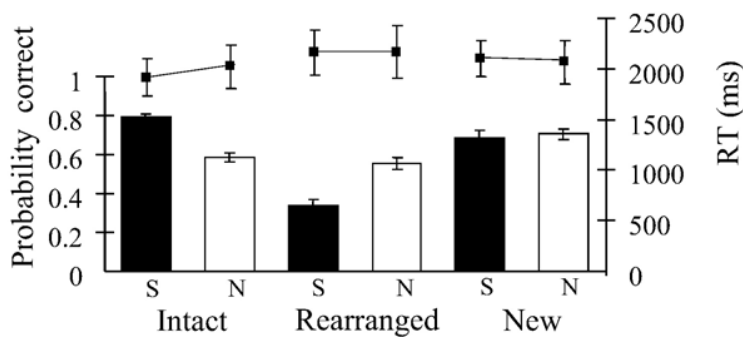

Fig. 2. Memory performance. Mean (and standard error) probability of a correct response (bars) and mean (and standard error) reaction times (lines) during the test phase, shown for intact, rearranged and new word pairs. Participants were required to respond old only to intact pairs. Responses are shown separately for the semantic (S) and non-semantic (N) conditions, indicating a clear enhancement in recognition of intact pairs for the semantic compared to non-semantic condition. contribution of familiarity. The probability of confidence ratings for all semantic hits was contrasted with the probability of confidence ratings for all non-semantic hits. The ANOVA with the factors of condition (semantic, non-semantic) and confidence rating (ratings 1-5) for correctly identified intact pairs revealed a main effect of condition $[F(1,14)=36.76 ; p<0.001]$, a main effect of confidence $[F(4,56)=11.23 ; p<0.001]$ and an interaction between confidence and condition $[F(4,56)=16.60 ; p<0.001]$, suggesting that the pattern of confidence ratings did vary across conditions. Importantly, a $t$-test confirmed that there was indeed an increase in confidence for the semantic compared to the nonsemantic condition at the middle range confidence category 4 $(t(14)=2.43, p=0.029)$. This difference did not reach significance for confidence categories 2 and 3 , but given the increase in category 4 ratings, it is likely that there was less opportunity for ratings at categories 2 and 3. Nonetheless, this suggests that semantic pairs did attract more ratings in the middle range, particularly at higher confidence levels, reflecting stronger familiarity for semantic pairs. Finally, a $t$-test contrasting the 'high confidence ratings' also reveals increased confidence ratings for semantic pairs $(t(14)=6.32, p<0.001)$. Since familiarity as well as recollection is likely to contribute to 'high confidence ratings' it is difficult to determine which process is accountable for this difference. Nevertheless, stronger familiarity for semantic pairs is associated with a shift in confidence ratings towards higher values and would predict the observed increase in high confidence ratings.

\section{Electrophysiological data}

Grand average ERPs for correct responses to intact and new word pairs are shown for the semantic condition in Fig. 3, and for the non-semantic condition in Fig. 4. The mean number of trials contributing to the intact and new waveforms were 32 and 29 for semantic, and 24 and 31 for non-semantic. Figs. 3 and 4 show that the ERPs evoked in both semantic and non-semantic conditions are more positive-going for intact compared to new word pairs from around $300 \mathrm{~ms}$. The old/new effects are initially bilaterally distributed and largest over frontal electrodes for the semantic condition, with a more central focus for the non-semantic condition. From around $600 \mathrm{~ms}$, however, the positive shift for intact pairs exhibits a clear left parietal maximum in both conditions. The intact and new waveforms converge around $900 \mathrm{~ms}$, but differences appear to remerge from around $1200 \mathrm{~ms}$ onwards. To characterize the pattern of old/new effects for each condition, average voltages were calculated over four consecutive epochs (300-600 ms, 600-900 ms, 900-1200 ms, 1200-2000 ms), at 4 separate locations, representing average activity over 3 electrodes: left-frontal (LF: F1, F3, F5), right-frontal (RF: F2, F4, F6), left-parietal (LP: CP1, CP3, CP5) and right-parietal (RP: CP2, CP4, CP6), as illustrated in Fig. 5A.

Initial high-level analyses were designed to identify whether old/new effects varied across conditions and epochs, using ANOVA with the factors of condition (semantic, non-semantic), epoch (300-600 ms, 600-900 ms, 900-1200 ms, 1200-2000 ms), old/new (intact, new), location (frontal, parietal) and hemisphere (left, right). Results revealed significant ERP old/new effects; a main effect of old/new $[F(1,14)=8.41, p=0.012]$, a two-way interaction between old/new and location $[F(2.67,37.35)=7.5$, $p=0.001]$ and three three-way interactions between condition, epoch and old $/$ new $[F(2.18,30.5)=5.01, p=0.011]$, epoch, old $/$ new 


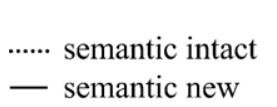

NP1
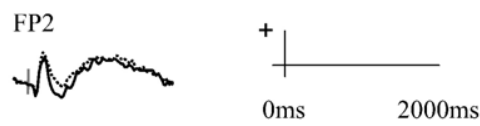

F8

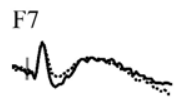<smiles>C=CCCC(C)C</smiles><smiles>[R]C(C)(C)COCCC</smiles>

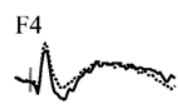

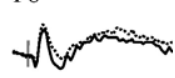

T7<smiles>C=C=CCCCCCC</smiles><smiles>CC#CC=CCC</smiles><smiles>C=CC=CCCC</smiles>

$\mathrm{C} 4$<smiles>CCCCCCC</smiles>

T8

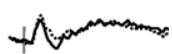

CP4

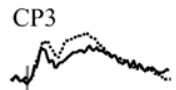<smiles>C=Cc1ccc(C)cc1</smiles>

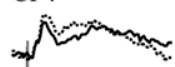

TP8

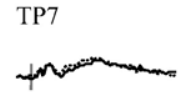

PO3
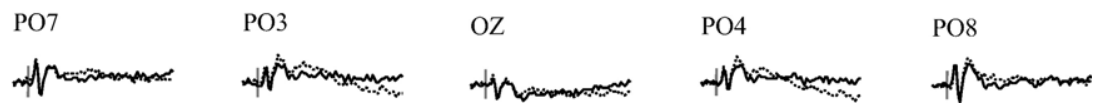

Fig. 3. Grand average ERPs for semantic word pairs. ERP waveforms are shown from 22 electrodes, displayed as if looking down onto the top of the head. ERPs are shown for correct responses to intact (thin) and new (thick) word pairs, differences between the waveforms revealing a characteristic pattern of ERP old/new effects, with an mid-frontal ERP effect followed by a strong left parietal effect.

and location $[F(2.50,34.93)=4.3, p=0.016]$ and old $/$ new, location and hemisphere $[F(1,14)=4.75, p=0.047]$. The presence of significant old/new interactions involving factors of both epoch and location strongly suggests that a changing pattern of old/new effects exists over the four epochs, varying across the semantic and non-semantic conditions, and varying across frontal and parietal locations.
To further investigate the pattern of old/new effects, a second level of analysis was performed on the data from each epoch, using ANOVA with the factors of old/new (intact, new), location (frontal, parietal) and hemisphere (left, right). This analysis aimed to demonstrate whether old/new effects were present within each epoch. The results are shown in Table 1; significant effects involving the factor of old/new are present during the 300-600 ms

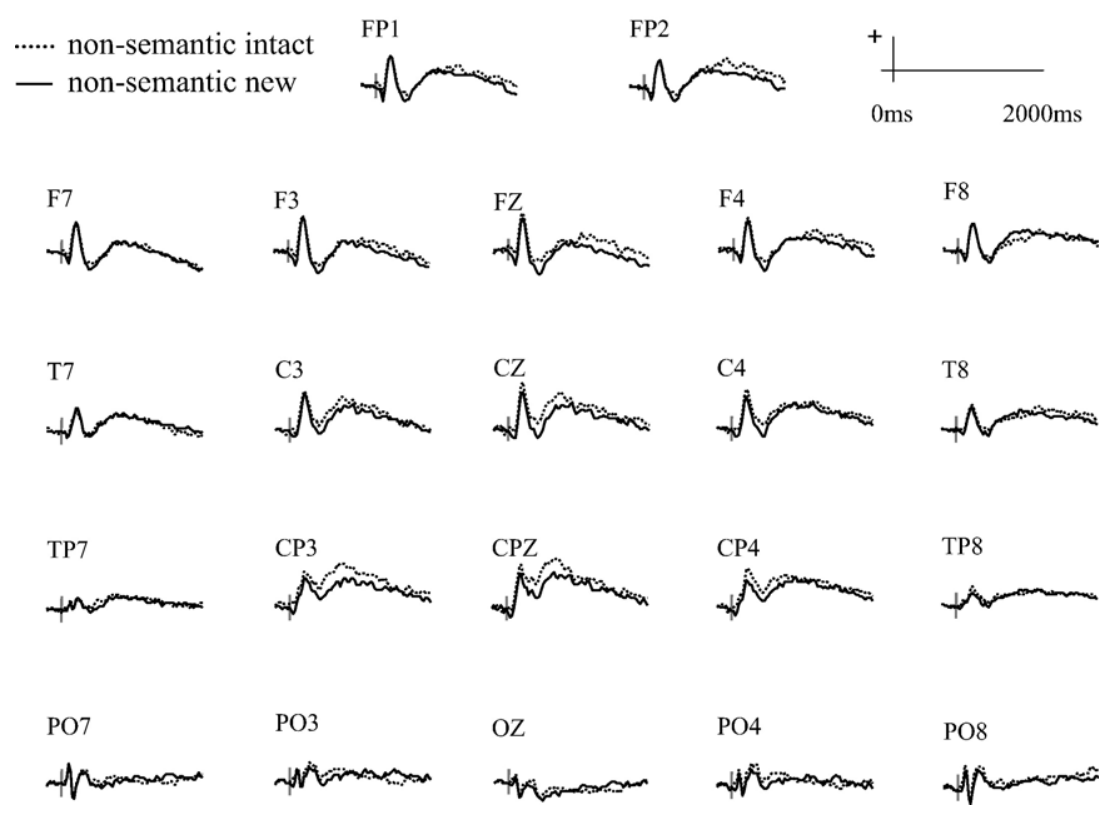

Fig. 4. Grand average ERPs for non-semantic word pairs. ERP waveforms are shown from 22 electrodes, displayed as if looking down onto the top of the head. ERPs are shown for correct responses to intact (thin) and new (thick) word pairs, exhibiting a different pattern of ERP old/new effects compared to those seen for semantic word pairs. While a clear left parietal effect is present, there is little evidence of an early mid-frontal ERP effect. 
A

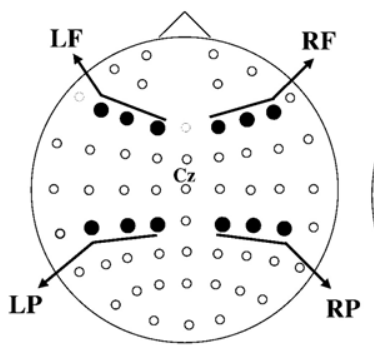

B

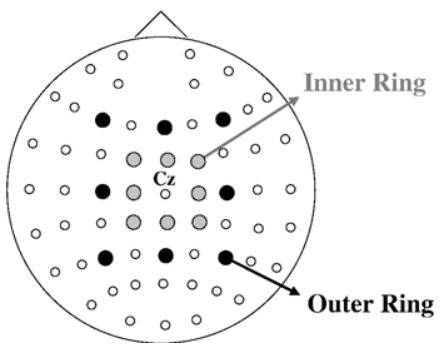

Fig. 5. Schematic of the recording montage and analysis strategy. Each figure depicts all 61 EEG electrodes, shown as if looking down onto the top of the head, with the nose at the front. Electrode $C z$ is identified for orientation; all electrodes are listed in the Materials and methods. Filled electrodes are used in specific analyses. (A) ERP analyses investigating the mid-frontal ERP old/ new effect and left parietal old/new effects were performed over four regions, each representing average activity over 3 electrodes: left-frontal (LF: F1, F3, F5), right-frontal (RF: F2, F4, F6), left-parietal (LP: CP1, CP3, CP5) and right-parietal (RP: CP2, CP4, CP6). (B) ERP analysis investigating N400 effects included electrodes located in two rings, each containing 8 electrodes (Inner Ring: FCZ, FC2, C2, CP2, CPZ, CP1, C1, FC1; Outer Ring: FZ, F4, C4, P4, PZ, P3, C3, F3), allowing the central maximum of the effect to be identified.

and 600-900 ms epochs, but not for the later two time windows. As Table 1 shows, in both of the early epochs the factor of old/new interacts with location (albeit marginally significant for the first epoch), suggesting that the pattern of old/new effects varies at frontal and parietal electrodes. This pattern of results is consistent with the presence of an early effect over frontal scalp, and a later effect (which varies by hemisphere) over parietal scalp. To demonstrate that this is indeed the case, and to investigate whether these old/new effects differ across semantic and non-semantic conditions, a final set of analysis was performed separately at frontal and parietal locations during the $300-600 \mathrm{~ms}$ and $600-$ $900 \mathrm{~ms}$ epochs. These data are highlighted in Fig. 6.

Analysis of the data from 300-600 ms employed ANOVA with the factors of condition (semantic, non-semantic), old/new (intact, new) and hemisphere (left, right). Analysis at frontal electrodes revealed a significant main effect of old/new $[F(1,14)=20.32$, $p<0.001]$, and an interaction between old/new and condition $[F(1,14)=6.30, p=0.025]$. As Fig. 6 shows, old/new effects are present over frontal scalp sites from 300 to $600 \mathrm{~ms}$, and these effects are significantly larger for the semantic condition. This result was confirmed with an additional focused analysis (collapsed across frontal electrodes) that directly compared the magnitude of the old/new difference across conditions $(t(14)=2.45, p=0.028)$. By contrast, analysis at parietal electrodes revealed a significant effect of old/new $[F(1,14)=33.57, p<0.001]$ but no interactions. This pattern of results is important; it reveals differences in the old/ new effects exhibited by semantic and non-semantic conditions only at frontal electrodes. The topographic distribution of the old/ new effects is illustrated in Fig. 7, highlighting a bilaterally distributed old/new effect over frontal scalp for the semantic condition only, and a more posterior effect that is present for both the semantic and non-semantic conditions.

Analysis of the data from 300-600 ms revealed significant old/ new differences at centro-parietal locations, though these effects did not differ in the semantic and non-semantic conditions. This old/new difference is particularly visible in Fig. 7 for the non-semantic condition, where there is little sign of additional overlapping frontally distributed effects. Examination of the waveforms (see Fig. 4) suggests that, in this case, the difference appears to be restricted to a window from $300-500 \mathrm{~ms}$ and is largest over central electrodes. This pattern of old/new differences is consistent with a modulation of the N400 component, a negative going peak (maximal over central-parietal electrodes between 300 and $500 \mathrm{~ms}$ post-stimulus) which is known to vary with the ease with which the meaning of words can be integrated into a given context (for review see Kutas and Van Petten, 1994). To be clear, we distinguish here between the N400 component (the negative going peak) and the N400 old/new effect (a modulation of the N400 component). Given the pattern of data shown in Figs. 6 and 7, it seems likely that the small frontally distributed old/new differences seen for the non-semantic condition most likely reflects residual spread of activity from the N400 old/ new effects seen for intact pairs (where the words have already been integrated) compared to new pairs (where integration is required).

Based on the pattern of effects seen at central and frontal electrodes, two possible interpretations of the data exist. First, both the semantic and non-semantic conditions are associated with activity over frontal electrodes that reflect spread of activity from the N400 old/new effects, but that for the semantic condition this is overlaid with additional mid-frontal activation from a second more frontally distributed generator. Second, both the semantic and nonsemantic conditions are associated with activity over frontal electrodes that reflect spread of activity from the N400 old/new effects, and the increased old/new effect at frontal electrodes in the semantic condition simply results from a spread of a stronger N400 old/new component, with no additional purely mid-frontal activation present.

To discriminate between the two possibilities outlined above, additional analyses were performed on data from $300-500 \mathrm{~ms}$, over two rings of electrodes that capture the distribution of the N400 component (see Fig. 5B). ANOVA was performed with factors of condition (semantic, non-semantic), old/new (intact, new), ring (inner, outer) and site (FCZ, FC2, C2, CP2, CPZ, CP1, C1, FC1 and

Table 1

Statistical analyses of the old/new effects.

\begin{tabular}{|c|c|c|c|c|c|c|c|c|}
\hline & \multicolumn{4}{|c|}{$300-600 \mathrm{~ms}$} & \multicolumn{4}{|c|}{$600-900 \mathrm{~ms}$} \\
\hline & $F$ & $d f$ & $d f$ & $p$ & $F$ & $d f$ & $d f$ & $p$ \\
\hline Old/New & 29.9 & 1 & 14 & $<0.05$ & 9.39 & 1 & 14 & $<0.05$ \\
\hline Old/New by location & 3.81 & 1 & 14 & 0.071 & 6.94 & 1 & 14 & $<0.05$ \\
\hline $\begin{array}{l}\text { Old/New by location } \\
\text { by hemisphere }\end{array}$ & & & & n.s. & 7.80 & 1 & 14 & $<0.05$ \\
\hline
\end{tabular}

by hemisphere

\begin{tabular}{|c|c|c|c|c|c|c|c|c|}
\hline & 90 & 200 & & & 12 & 200 & & \\
\hline & $F$ & $d f$ & $d f$ & $p$ & $F$ & $d f$ & $d f$ & $p$ \\
\hline Old/New & & & & n.s & & & & n.s. \\
\hline Old/New by location & & & & n.s & & & & n.s. \\
\hline Old/New by location & & & & n.s & & & & n.s. \\
\hline
\end{tabular}

by hemisphere

Results of ANOVA with the factors of old/new (intact, new), location (frontal, parietal) and hemisphere (left, right) over four successive time windows. The data reveal significant effects involving the factor of old/new during the 300-600 ms and 600-900 ms epochs, but no significant effects for the later two time windows. In both of the early epochs, the old/new effects interact with location, suggesting that the pattern of old/new effects varies at frontal and parietal electrodes. 
A
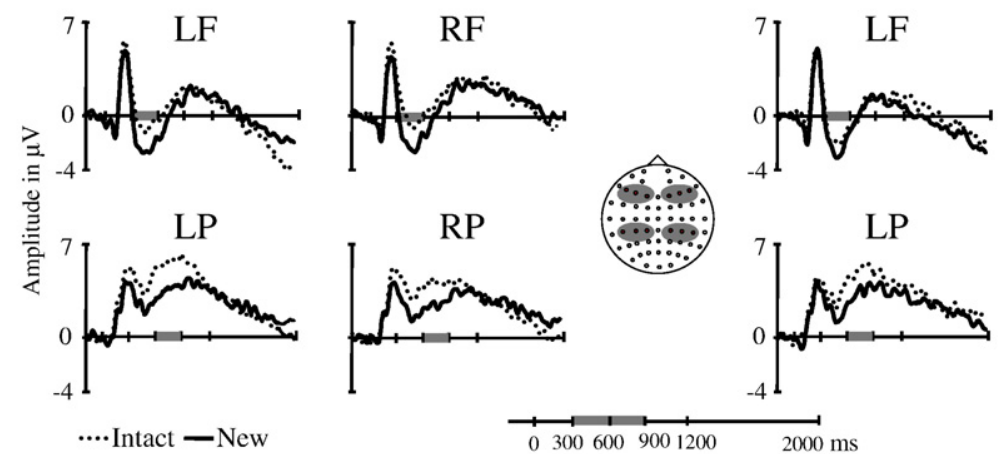
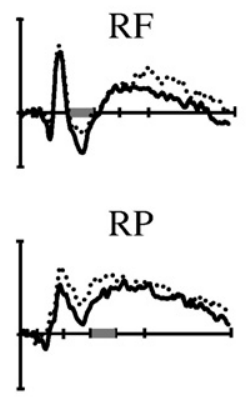

.... Intact - New
B

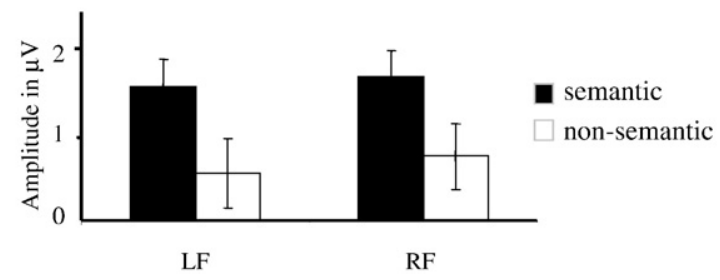

II.) Left parietal old/new effect

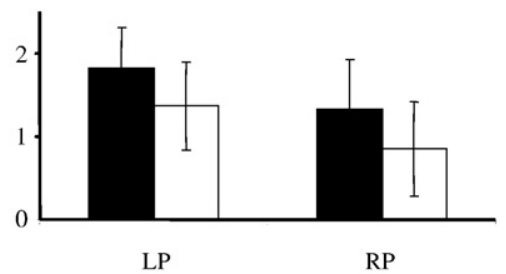

Fig. 6. Mid-frontal ERP old/new effect and left parietal old/new effects. The panels illustrate how the ERP correlates of familiarity and recollection are modulated by manipulating the semantic coherence of to-be-remembered word pairs. (A) Average ERP waveforms from left-frontal (LF: F1, F3, F5), right-frontal (RF: F2, F4, F6), left-parietal (LP: CP1, CP3, CP5) and right-parietal (RP: CP2, CP4, CP6) locations. Data are shown separately for semantic and non-semantic conditions, ERPs for intact and new waveforms shown in each case. Scale bars indicate the amplitude range and epoch length (with markers indicating the 300$600 \mathrm{~ms}$ and $600-900 \mathrm{~ms}$ windows). The mid-frontal ERP old/new effect (300-600 ms) is more pronounced for semantic compared to non-semantic conditions, while the left parietal old/new effect (600-900 ms) is similar in both cases. (B) Bar charts display the magnitude of the old/new effects (error bars show SE), averaged from the data shown in panel A. The mid-frontal ERP old/new effect (I) is significantly larger for the semantic compared to non-semantic conditions in the 300-600 ms epoch. By contrast, the parietal old/new effect (II) found from 600-900 ms reveals no reliable differences between the semantic and nonsemantic conditions.

FZ, F4, C4, P4, PZ, P3, C3, F3). Analysis revealed a significant effect of old/new $[F(1,14)=28.95, p<0.001]$, a two-way interaction between old/new and ring $[F(1,14)=27.51, p<0.001]$ and a threeway interaction between old $/$ new, ring and site $[F(4.1,58.3)=3.35$, $p=0.014]$. These analyses (a) confirm the characteristic distribution of the N400 component, maximal over a superior central ring of electrodes, (b) demonstrate that the N400 component is modulated by the old/new status of the test items and (c) reveal that this modulation of the N400 component does not differ between semantic and non-semantic conditions. Thus, as can be seen in Fig. 7, non-semantic word pairs reflect only a centrally distributed N400 old/new effect in the early time-window, whereas the semantic pairs reflect a comparable centrally distributed N400 old/new effect combined with an additional mid-frontal ERP old/new effect.

Data from 600-900 ms were analyzed using equivalent ANOVA with the factors of condition (semantic, non-semantic), old/new (intact, new) and hemisphere (left, right). These analyses revealed no significant old/new effects over frontal electrodes, but a significant main effect of old/new $[F(1,14)=25.25, p<0.001]$ and a significant interaction between old/new and hemisphere $[F(1,14)=4.67$, $p=0.049$ ] at parietal sites. As can be seen in Fig. 6, these findings reveal that old/new effects are present from 600-900 ms, exhibiting a left greater than right asymmetry characteristic of the left parietal old/new effect. Importantly, no old/new effects were present at frontal sites, and no differences were found between the old/new effects exhibited in the semantic and non-semantic conditions during the 600-900 ms epoch. The topographic distribution of the left parietal old/new effects is shown in Fig. 7 for both semantic and nonsemantic conditions.

\section{Topographic analyses}

The pattern of results described above strongly suggests differences in the ERP old/new effects found for semantic and non-semantic conditions during the $300-600 \mathrm{~ms}$ epoch. As Figs. 6 and 7 illustrate, an early mid-frontal ERP old/new effect is present for the semantic condition, and this is not apparent for the non-semantic condition. Further, the results suggest a change in activity over time for the semantic condition, from an early mid-frontal effect to a later left parietal effect. We performed additional topographic analysis to confirm that this finding reflects a genuine qualitative difference in the pattern of neural generators engaged over time. Analyses were performed for the semantic condition on difference waveforms (intact minus new) from the 300-600 and 600-900 ms epochs, using rescaled data (removing confounding effects of size). ANOVA with the factors of epoch, location and hemisphere revealed a significant three-way interaction $[F(1,14)=10.08, p<0.01]$. This analysis confirms that the early mid-frontal ERP old/new effect is statistically distinct from the later parietal effect, demonstrating the presence of two spatio-temporally dissociable memory processes. 


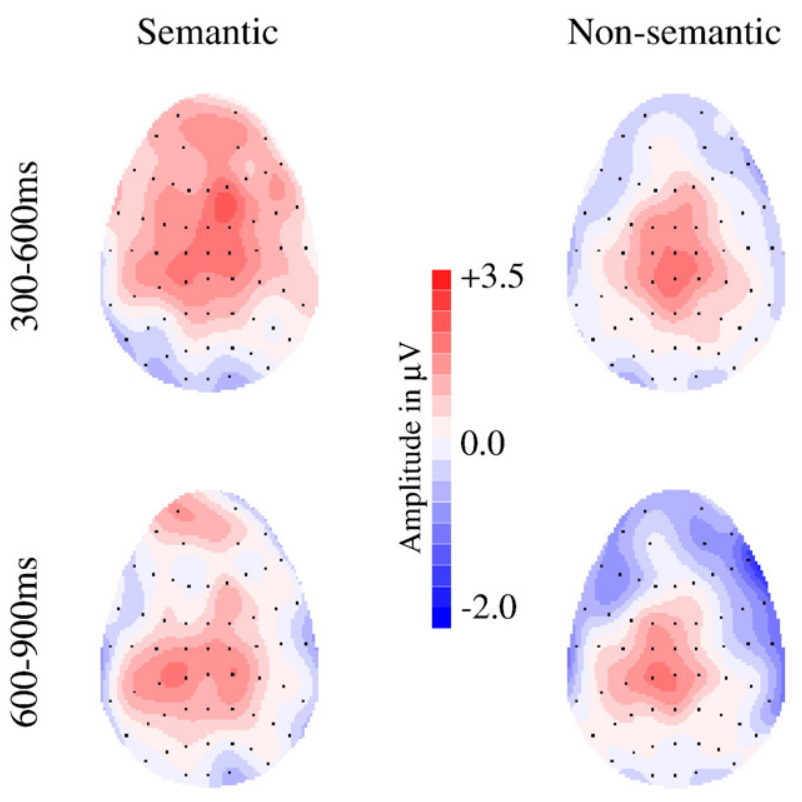

Fig. 7. Topographic distribution of the old/new effects. The maps illustrate the distribution of effects for semantic and non-semantic conditions. Each map represents the difference in activity between intact and new waveforms, averaged over time (300-600 ms and 600-900 ms). Maps are shown as if looking down onto the top of the head, with individual electrode locations indicated by black dots, and a scale bar shows amplitude range. The topographical distribution in the early 300-600 ms time window shows symmetric frontal activity in the semantic compared condition, compared to a more central parietal effect in the non-semantic condition. By contrast, in the 600-900 ms time window the ERP old/new effects show a left parietal distribution, with no significant differences between the semantic and nonsemantic conditions.

\section{Additional behavioral experiment}

The additional within-subjects PDP experiment was designed solely to provide estimates of recollection and familiarity, and the behavioral data are therefore reported in brief ${ }^{4}$. Statistical analysis of the exclusion data revealed the same pattern of results as

\footnotetext{
${ }^{4}$ Following the ERP 'exclusion' test, we originally carried out an 'inclusion' test using additional subjects, producing a between-subjects version of the PDP estimates of recollection and familiarity. While familiarity estimates increased from 0.48 to 0.71 for non-semantic compared to semantic pairs, estimates for recollection showed no equivalent increase, changing from 0.05 to 0.09 for non-semantic and semantic pairs respectively. Thus, these between-subject PDP data revealed a large increase in familiarity from non-semantic to semantic conditions $(0.23)$ but a considerably smaller difference in recollection $(0.04)$. This reflects the same pattern of results as the estimates reported for the withinsubjects data, which therefore replicate both the specific pattern of findings observed in the original ERP exclusion study, and the between-subject PDP estimates. The between-subjects data had the benefit of being (at least in part) from the same experiment as the reported ERP data, but the PDP estimates could not easily be subjected to statistical analysis, as it is unclear how performance in the inclusion task maps on to performance in the exclusion task. Nevertheless, statistical analysis of randomly paired inclusion and exclusion responses, as well as positively and negatively correlated pairs of inclusion and exclusion responses, all revealed the same result: significantly increased familiarity for semantic compared to nonsemantic conditions but no difference in recollection.
}

observed in the ERP experiment. ANOVA including the factors of relationship (semantic, non-semantic) and pairing (intact, impaired, new) revealed a significant main effect of pairing $[F(2,22)=18.87$, $p<0.001]$, no main effect of relationship [p>0.05], but a significant interaction between relationship and pairing $[F(2,22)=$ 14.22, $p<0.001]$. As was the case for the ERP experiment, intact pairs were better remembered when semantically related $(t(11)=$ 2.79, $p=0.018$ ) while rearranged pairs were more difficult to reject when semantically related $(t(11)=3.97, p=0.002)$.

The inclusion task also revealed differences in recognition performances for semantic and non-semantic word pairs. ANOVA with the factors of relationship (semantic, non-semantic) and pairing (intact, impaired, new) revealed a significant main effect of relationship $[F(1,11)=5.32, p=0.041]$ and a significant interaction between relationship and pairing $[F(2,22)=4.41, p=0.025]$. Intact pairs were better remembered when semantically related $(t(1)=2.11, p=0.059)$. In contrast to the exclusion task, rearranged pairs were associated with better performance for semantically related compared to unrelated pairs $(t(11)=3.91, p=0.002)$, as would be expected given the changes in task instruction.

\section{PDP estimates}

Based on the combined performance from the exclusion and inclusion studies, the process dissociation procedure (PDP) can be used to provide an estimate of the contribution of familiarity and recollection to performance. These data are illustrated in Fig. 8, showing that familiarity increased from 0.53 to 0.72 for nonsemantic compared to semantic pairs, while estimates for recollection remained stable at 0.26 and 0.24 for non-semantic and semantic pairs. Clearly, the PDP data exhibit a considerable increase in familiarity from non-semantic to semantic conditions (0.19) but only a small difference in recollection (0.02). The PDP estimates were subjected to statistical analysis and an ANOVA with the factors of condition (semantic vs. non-semantic) and estimate (familiarity vs. recollection), revealing a main effect of condition $[F(1,11)=7.35, p=0.02]$, a main effect of estimate $[F(1,11)=11.62$, $p<0.01]$ and a significant interaction between condition and estimate $[F(1,11)=14.23, p<0.01]$. Subsidiary analysis confirmed a statistically significant increase in familiarity from non-semantic to semantic conditions $(t(11)=6.13, p<0.001)$ and no significant difference in recollection $(t(11)=0.48, p>0.05)$. Thus, consistent

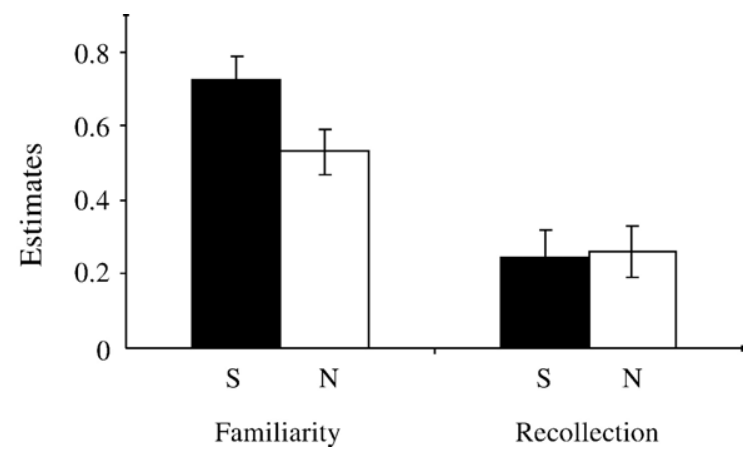

Fig. 8. PDP estimates of familiarity and recollection. PDP estimates (mean and standard error) of familiarity and recollection are shown for the semantic (S) and non-semantic $(\mathrm{N})$ condition. The estimates, which are derived from the behavioral inclusion and exclusion experiments, demonstrate a significant difference between semantic and non-semantic conditions in familiarity but not recollection. 
with the pattern of ERP effects, behavioral estimates suggest that episodic retrieval is modulated by the manipulation of semantic vs. non-semantic word pairs, and that this is driven primarily by changes in familiarity rather than recollection.

\section{Discussion}

We investigated the functional and neural interaction between episodic and semantic memory in normal cognition. By manipulating whether word pairs were categorically related, we demonstrated that associative recognition memory is enhanced for semantically coherent information. Second, we asked if this interaction produced changes in recollection or familiarity. To our surprise, the data revealed that the influence of semantic memory was to modulate the extent of familiarity based retrieval, leaving recollection relatively unaffected. In short, our data provide novel evidence for an interaction between semantic and episodic memory, demonstrating that the semantic organization of information influences the engagement of episodic memory by modulating familiarity based retrieval.

\section{Convergent evidence that manipulating semantic memory modulates familiarity}

Our results are particularly compelling because they reflect convergent behavioral and electrophysiological evidence. Estimates derived from the process dissociation procedure (Jacoby, 1991) revealed a considerable increase in familiarity, alongside a negligible change in recollection. While the behavioral findings are indirect, the neuroimaging data provide a direct record of the underlying neural activity associated with performance. As would be expected on the basis of previous findings (Donaldson and Rugg, 1999), our data provide clear evidence of significant left parietal old/new effects during associative recognition memory (see Figs. 6 and 7). Importantly, however, there was no evidence for a significant difference in the left parietal old/new effect elicited by semantic and non-semantic word pairs; consistent with the behavioral data, ERPs revealed comparable levels of recollection. By contrast, the semantic and non-semantic conditions were dissociable on the basis of the mid-frontal ERP old/new effect, which was only present for the semantic condition. The ERP findings are particularly clear; the semantic and non-semantic conditions exhibited a selective modulation of the mid-frontal ERP old/new effect, suggesting that recognition of the semantic word pairs was enhanced due to an increase in familiarity.

Although the PDP and ERP findings both point towards the conclusion that semantic coherence influences familiarity rather than recollection, it is important to recognize that the reasons for reaching this conclusion are quite different in each case. While the ERP findings are restricted to analysis of the intact pairs, the PDP estimates stem primarily from performance on the rearranged pairs (the likelihood of hits and false alarms to this class of stimuli during the inclusion and exclusion tasks, respectively). In some respects, the difference between these two measures is a strength; the two findings provide genuinely independent (yet convergent) evidence using methods that rely on very different assumptions. Nonetheless, drawing any direct correspondence between the ERP and PDP data relies on a strong assumption, namely that the selective familiarity advantage for rearranged pairs from the semantic vs. non-semantic condition seen in the PDP data also holds for the intact pairs in the ERP data.
To address this issue and provide a more direct link between the behavioral and electrophysiological evidence, we carried out an additional examination of the confidence judgements made during the ERP task. Recollection is associated mostly with high confidence judgements, whereas familiarity typically covers a range of confidence judgements (Yonelinas, 1997; Yonelinas, 2001). If, as we believe, familiarity shows an increased contribution to intact semantic pairs compared to non-semantic pairs, this should be reflected in the pattern of confidence judgements. Consistent with this view, analysis of the confidence ratings revealed differences between semantic and non-semantic pairs for confidence ratings which are typically assumed to reflect responses based on familiarity. This finding adds weight to our conclusion, providing added behavioral evidence that the putative change in familiarity based responding is evident for both the rearranged and intact pairs. Given this confidence analysis, the ERP findings alone provide strong evidence for an increase in familiarity for the semantic, compared to non-semantic, condition.

\section{Unitization, bias and task difficulty}

One potential objection to our findings is that, traditionally, associative recognition tests are thought to require recollection (Atkinson and Juola, 1974; Mandler, 1980, 1991), with familiarity playing little or no role in performance. Recent evidence suggests, however, that this is not always the case; familiarity can sometimes contribute to associative recognition. For example, Yonelinas et al. (1999) demonstrated that familiarity can play a significant role in associative recognition if the separate elements of a stimulus are unitized into a single larger representation (e.g., complex stimuli such as faces can be familiar when processed as a whole, but not when processed in parts). Our findings are consistent with this view; enhanced performance and increased familiarity for the semantically coherent stimuli may reflect the benefits of unitization. We are cautious about interpreting our data in this way, however, because the extent and limits of unitization are not well understood to date. For example, it is unclear what kinds of stimulus relationships support unitized representations, or whether a brief learning phase (as employed in the present experiment) would allow unitization of item-item associations to occur (see Mayes et al., 2004, for argument that unitization is sufficient but not essential for an engagement of familiarity in associative recognition). Finally, we note that a unitization account need not sit in opposition to our interpretation of the data; rather, the unitization hypothesis may offer an explanation of the way in which semantic memory influences episodic retrieval.

Our data are, of course, open to alternative interpretations. Here we consider the possibility that the findings reflect little more than changes in either task difficulty or response bias. Were the lower levels of performance in the non-semantic condition, and thus the lower estimates of familiarity, simply due to increased task difficulty? Task difficulty is notoriously hard to define a priori, and is typically introduced as an a posteriori explanation of changes in performance. In the present case, although performance is poorer in the non-semantic condition overall (consistent with a change in difficulty), reaction times are not significantly different in the semantic and non-semantic conditions (suggesting no overall change in difficulty). More importantly, both the behavioral and ERP findings suggest that the manipulation of semantic coherence had a selective effect; unlike familiarity, recollection did not vary across condition. By contrast, a task difficulty account would 
predict changes in both familiarity and recollection, and not a selective change in familiarity.

An alternative interpretation of our data is that the ERP findings reflect little more than the influence of response bias. As reported above, the behavioral data reveal a high response bias for semantic pairs and a low response bias for non-semantic pairs. How might bias influence the ERPs measured at retrieval? Perhaps the most straightforward view would be that changes in bias should directly influence the probability of responding old on the basis of familiarity (a more liberal bias leading to an increased likelihood of items being accepted as old on the basis of a lower level of familiarity). Our data do, superficially, fit such a simple bias account; the more liberal bias found for the semantic condition was associated with better performance and an increase in familiarity. Thus, assuming that changes in response bias exhibit more influence on familiarity than recollection, encountering a semantic relationship between the category name and word pair may have resulted in a relaxed criterion for responding 'old' overall, producing a change in the size of the mid-frontal old/new effect. From this perspective, the semantic condition is seen as being associated with increased familiarity based responding (as indexed by the mid-frontal old/new effect), but this is simply a consequence of changes in response bias.

While a response bias account is plausible, we find it difficult to sustain. Our data do not reveal a change in bias alone; the behavioral results show both a change in bias and a change in discriminability, with significantly higher discrimination in the semantic compared to non-semantic condition. Moreover, the behavioral results for the semantic and non-semantic conditions show no significant differences in false alarm rate. A full account of our data would therefore require an explanation that goes beyond response bias. Moreover, it is clear from previous findings that ERPs are sensitive to changes in response bias at frontal recording sites, but that the neural correlates of response bias may be distinct from those of familiarity, with the former seen only in responses to new items (cf. Johansson et al., 2004), or occurring in opposite directions for old and new items (cf. Windmann et al., 2002). In the present case, careful examination of the data reveals that the change in the pattern of mid-frontal old/new effects across semantic and non-semantic conditions is driven by the old conditions (see Fig. 6). Additional analysis comparing the magnitude of activity across the two correct rejection waveforms from 300 to $600 \mathrm{~ms}$ at frontal electrodes revealed no significant difference between the semantic and nonsemantic conditions (means of $-2.1 \mu \mathrm{V}$ and $-1.9 \mu \mathrm{V}$, respectively; $F(1,14)=0.13, p>0.05$ ). If the mid-frontal old/new effect was modulated by bias, this would be expected to be evident in the ERPs to correct rejections. Thus, although differences in bias are present across conditions, and this could theoretically underlie the change in familiarity that is seen, we do not favor a bias account. Finally, we note that this does not matter a great deal for our central conclusion, which is that the change from semantic to non-semantic conditions is associated with an increase in familiarity, rather than recollection. Whether this turns out to be associated with changes in unitization, bias, task difficulty, or other factors, remains an interesting question.

\section{Dissociating the mid-frontal ERP old/new effect and N400 old/new effects}

One important aspect of our data is the clear dissociation between the mid-frontal ERP old/new effect and the N400 old/new effect. The N400 component is typically seen in studies of language comprehension, and reflects the difficulty with which the meaning of words can be integrated with the current context (for review see Kutas and Van Petten, 1994). Given our manipulation, overall differences in the N400 component are to be expected in the semantic and non-semantic conditions (with a larger N400 for non-semantic compared to semantic pairs). Of more concern was the possibility that differences in the old/new effects across condition might simply have reflected a change in the pattern of N400 old/new effects. Modulation of the N400 component elicited by intact and new pairs (i.e., N400 old/new effects) are reasonable, as semantic integration for intact pairs (where words have already been integrated during study) is easier compared to new pairs (where additional integration is required). It was, therefore, critical that the observed N400 old/new effect did not vary across the semantic and non-semantic conditions. Any such difference would have suggested that our manipulation merely influenced the ease of semantic integration and not episodic retrieval itself.

The present findings are, consequently, significant in ruling out interpretations of the mid-frontal ERP old/new effect as nothing more than a reduction of the N400 component (e.g., as suggested by Yovel and Paller, 2004). In the present data, changes in the N400 component (i.e., N400 old/new effects) occurred in both semantic and non-semantic conditions, exhibiting a temporal overlap with the mid-frontal ERP old/new effect. The mid-frontal ERP old/new effect was, however, only present in the semantic condition, and thus cannot reflect a modulation of the N400 component per se. By contrast, the ERP data are, in principle, consistent with the possibility that the mid-frontal ERP old/new effect reflects summation of the N400 old/new effect with an anterior fronto-polar effect (cf. Curran and Dien, 2003). This anterior fronto-polar effect is, however, associated with visual (perceptual) priming, and there seems little reason to expect differences in visual priming across our semantic and non-semantic conditions. Regardless, the present data clearly suggest that the mid-frontal ERP old/new effect is driven by a different neural source than that which underlies the N400 component.

While we interpret the mid-frontal ERP old/new effect as reflecting familiarity, others have suggested that it reflects conceptual priming. One source of support for this view is the idea discussed above, that the mid-frontal ERP old/new effect is actually produced by an N400 reduction (Yovel and Paller, 2004), which our data clearly militate against. In addition, in the current context, a conceptual priming account would predict differences in the magnitude of the mid-frontal old/new effect between the semantic and non-semantic new pairs - the former are preceded by a semantically related category cue, but the later are not. Examination of the ERPs for correctly rejected new pairs revealed no differences over mid-frontal electrodes however, suggesting that the mid-frontal old/new effect is not elicited by conceptual priming. Moreover, evidence from normal (Wolk et al., 2004) and amnesic (Olichney et al., 2000) participants suggests that conceptual priming modulates the N400 component, separate from the mid-frontal ERP old/new effect. There are also clear examples of dissociations between conceptual priming and episodic memory retrieval using other neuroimaging methods (Donaldson et al., 2001) and neuropsychological data (Levy et al., 2004). We therefore favor the view that, at least for verbal material, the conscious experience of familiarity and the unconscious activation associated with conceptual priming are both functionally and neurally distinct. 


\section{Multiple interactions between semantic and episodic memory}

Our findings stand in striking contrast to results from Levels Of Processing studies, which typically reveal an interaction between episodic and semantic memory that is driven largely by a modulation of recollection. For example, Rugg et al. (1998; see also Rugg et al., 2000) measured ERP old/new effects during an LOP study (sentence generation vs. alphabetic judgement), and revealed a modulation of the left parietal effect but no change in the mid-frontal ERP old/new effect. In LOP studies the way in which information is processed is manipulated during learning. By contrast, here we manipulated the type of information that had to be remembered, while holding processing demands constant. Thus, while changes in semantic processing during learning lead to a modulation of recollection, it appears that changes in the semantic coherence of material results in a modulation of familiarity. Whether this result extends to other manipulations of semantic memory will be of considerable interest. Regardless, it is clear that the way in which semantic memory is manipulated has a critical impact on the interaction between episodic and semantic memory.

Neuropsychological and neuroimaging studies suggest that semantic knowledge is organized as categorical representations (Okada et al., 2000; for a review see Capitani et al., 2003). This type of organization is observable neuroanatomically (Warrington and McCarthy, 1983; Laiacona et al., 1998) and affects psychological processing, as demonstrated in semantic priming experiments (Collins and Loftus, 1975). Our findings suggest that this organization of semantic knowledge has a significant impact on the normal functioning of episodic memory retrieval. Our data go further, however, to suggest a mechanism that underlies this interaction in healthy subjects, namely familiarity. In general, familiarity can be interpreted as a consequence of the functional overlap amongst representations. We believe that familiarity arises when subjects become exposed to an item that matches (or largely overlaps with) previously stored representations. As mentioned above, semantic memory is categorically organized and items belonging to the same category are thought to share overlapping representations. By this view, it is plausible that the process of familiarity is particularly sensitive to the presence or absence of semantic relations between stimuli per se; whether it is the specific cueing of semantic categories employed here that is critical remains to be seen.

Finally, we suggest that future studies should examine the consequences that different types of manipulation of semantic memory have on behavior. We distinguish between manipulations of semantic content, which are inherent to the stimulus material and their representations, and manipulation of semantic context, which are dependent on the way stimuli are processed. In general, this difference might explain why processing manipulations in LOP studies (semantic deep vs. shallow encoding process) lead to recollection based interactions, while changes in material as applied in the current study (categorically related vs. unrelated word pairs) facilitate familiarity based interactions. We believe that these findings have important practical implications, for example, in relation to alleviating the memory impairments associated with aging. Processing manipulations require the active and intentional use of internal mental strategies, whereas material manipulations are external and independent from the engagement of strategies. As old people exhibit particular difficulty in strategic processing, manipulating the semantic organization of to-be-remembered material might prove a more effective way to enhance their episodic remembering.

\section{Conclusion}

In real life, when required to remember an important piece of information, one may strategically engage in elaborate thought to increase the likelihood of recollecting the information later. More often, however, memory operates without such intent. In this case, one of the important factors in determining whether information is remembered or forgotten is the characteristics of the information itself. Some of the information that we are required to remember maps onto our semantic knowledge about the world; it is coherently organized around a semantic theme or category. Alternatively, information can be relatively incoherent, without obvious relationship to existing knowledge, or any clear theme or category structure. As we have shown here, the nature of to-be-remembered information is important for how we remember; when information contains a coherent semantic structure, we are better able to remember it. Unlike changing the way in which information is processed, however, making information more semantically coherent simply causes it to become more familiar.

\section{Acknowledgments}

Thanks to Jim Bednar and anonymous reviewers for helpful comments. A.G. is supported by a DTC studentship funded by the EPSRC. D.I.D. is supported by a grant from the BBSRC.

\section{References}

Allan, K., L. Wilding, E., Rugg, M.D., 1998. Electrophysiological evidence for dissociable processes contributing to recollection. Acta Psychol. 98 (2-3), 231-252.

Atkinson, R.C., Juola, J.F., 1974. Search and decision processes in recognition memory. In: Krantz, D.H., Atkinson, R.C. (Eds.), Contemporary Developments in Mathematical Psychology: I. Learning, Memory and Thinking. W.H. Freeman, Oxford, England.

Azmimian-Faridani, N., Wilding, E.L., 2004. An event-related potential study of the revelation effect. Psychon. Bull. Rev. 11, 926-931.

Capitani, E., Laiacona, M., Mahon, B., Caramazza, A., 2003. What are the facts of category-specific deficits? A critical review of the clinical evidence. Cogn. Neuropsychol. 20 (3/6), 213-261.

Collins, A., Loftus, E.A., 1975. Spreading-activation theory of semantic processing. Psychol. Rev. 82 (6), 407-428.

Craik, F.I., Lockhart, R.S., 1972. Levels of processing: a framework for memory research. J. Verbal Learn. Verbal Behav. 11 (6), $671-684$.

Curran, T., 1999. The electrophysiology of incidental and intentional retrieval: ERP old/new effects in lexical decision and recognition memory. Neuropsychologia 37 (7), 771-785.

Curran, T., 2000. Brain potentials of recollection and familiarity. Mem. Cogn. 28 (6), 923-938.

Curran, T., Dien, J., 2003. Differentiating amodal familiarity from modalityspecific memory processes: an ERP study. Psychophysiology 40, 979-988.

Delis, D.C., Kramer, J.H., Kaplan, E., et al., 1987. California Verbal Learning Test Manual (Research Edition). Psychological Corporation/ Harcourt Brace, San Antonio, TX.

Donaldson, D.I., Rugg, M.D., 1999. Event-related potential studies of associative recognition and recall: electrophysiological evidence for context dependent retrieval processes. Cogn. Brain Res. 8 (1), 1-16. 
Donaldson, D.I., Petersen, S.E., Buckner, R.L., 2001. Dissociating memory retrieval processes using fMRI: evidence that priming does not support recognition memory. Neuron 31 (6), 1047-1059.

Donaldson, D.I., Allan, K.A., Wilding, E.L., 2002. Electrophysiological investigations of explicit memory retrieval. In: Wilding, E.L., Parker, A., Bussey, T. (Eds.), The Cognitive Neurosciences. Psychology Press.

Dosher, B.A., 1984. Discriminating preexperimental (semantic) from learned (episodic) associations: a speed-accuracy study. Cogn. Psychol. 16 (4), 519-555.

Gobet, F., 1998. Expert memory: a comparison of four theories. Cognition $115-152$.

Graham, K.S., Simons, J.S., Pratt, K.H., Patterson, K., Hodges, J.R., 2000. Insights from semantic dementia on the relationship between episodic and semantic memory. Neuropsychologia 38 (3), 313-324.

Jacoby, L.L., 1991. A process dissociation framework: separating automatic from intentional uses of memory. J. Mem. Lang. 30 (5), $513-541$.

Jasper, H., 1958. The ten-twenty system of the international federation. Electroencephalogr. Clin. Neurophysiol. (10), 37-75.

Johansson, M., Mecklinger, A., Treese, A.-C., 2004. Recognition memory for emotional and neutral faces: and event-related potential study. J. Cogn. Neurosci. 16, 1840-1853.

Johnson, R.J., 1995. Event-related potential insights into the neurobiology of memory systems. In: Boller, F., Grafman, J. (Eds.), Handb. Neuropsychol., vol. 10, pp. 135-163.

Kucera, H., Francis, W.N., 1967. Computational Analysis of Present-Day American English. Brwon Univ. Press, Providence, RI.

Kutas, M., Van Petten, C.K., 1994. Psycholinguistic electrified: eventrelated brain potentials during sentence processing. In: Gernsbacher, M.A. (Ed.), Handbook of Psycholinguistics. Academic Press, San Diego, pp. 83-143.

Laiacona, M., Barbarotto, R., Capitani, E., 1998. Semantic category dissociations in naming: is there a gender effect in Alzheimer's disease? Neuropsychologia 36 (5), 407-419.

Levy, D.A., Stark, C.E.L., Squire, L.R., 2004. Intact conceptual priming in the absence of declarative memory. Psychol. Sci. 15 (10), 680-686.

Mandler, G., 1980. Recognizing: the judgment of previous occurrence. Psychol. Rev. 87 (3), 252-271.

Mandler, G., 1991. Your face looks familiar but I can't remember your name: a review of dual process theory. In: Murdock, B.B., Hockley, W.E., Lewandowsky, S. (Eds.), Relating Theory and Data: Essays on Human Memory in Honor of Bennet B. Murcdock. Lawrence Arlbaum Associates, pp. 207-255.

Mayes, A.R., Montaldi, D., 2001. Exploring the neural bases of episodic and semantic memory: the role of structural and functional neuroimaging. Neurosci. Biobehav. Rev. 25, 555-573.

Mayes, A.R., Holdstock, J.S., Isaac, C.L., Montaldi, D., Grigor, J., Gummer, A., Cariga, P., Downes, J.J., Tsivilis, D., Gaffan, D., Norman, K.A., 2004. Associative recognition in a patient with selective hippocampal lesions and relatively normal item recognition. Hippocampus 14 $763-784$.

McCarthy, G., Wood, C.C., 1985. Scalp distributions of event-related potentials: an ambiguity associated with analysis of variance models. Electroencephalogr. Clin. Neurophysiol./Evoked Potentials Section 62 (3), 203-208.

Mulligan, N., Hirshman, E., 1995. Speed-accuracy trade-offs and the dual process model of recognition memory. J. Mem. Lang. 34 (1), $1-18$.

Nessler, D., Mecklinger, A., Penney, T.B., 2001. Event related brain potentials and illusory memories: the effects of differential encoding. Cogn. Brain Res. 10 (3), 283-301.

Norman, K.A., Schacter, D.L., 1997. False recognition in younger and older adults: exploring the characteristics of illusory memories. Mem. Cogn. 25 (6), 838-848.

Okada, T., Tanaka, S., Nakai, T., Nishizawa, S., Inui, T., Sadato, N.,
Yonekura, Y., Konishi, J., 2000. Naming of animals and tools: a functional magnetic resonance imaging study of categorical differences in the human brain areas commonly used for naming visually presented objects. Neurosci. Lett. 296 (1), 33-36.

Olichney, J.M., Van Petten, C., Paller, K.A., Salmon, D.P., Iragui, V.J., Kutas, M., 2000. Word repetition in amnesia: electrophysiological measures of impaired and spared memory. Brain 123 (9), $1948-1963$.

Paller, K.A., Kutas, M., 1992. Brain potentials during memory retrieval provide neurophysiological support for the distinction between conscious recollection and priming. J. Cogn. Neurosci. 4 (4), 375-391.

Roediger, H.L., Bergman, E.T., 1998. The controversy over recovered memories. Psychol. Public Policy Law 4, 1091-1109.

Roediger, I.H.L., McDermott, K.B., 1995. Creating false memories: remembering words not presented in lists. J. Exper. Psychol., Learn., Mem., Cogn. 21 (4), 803-814.

Rugg, M.D., 1995. Memory and consciousness: a selective review of issues and data. Neuropsychologia 33 (9), 1131-1141.

Rugg, M.D., Mark, R.E., Walla, P., Schloerscheidt, A.M., Birch, C.S., Allan, K., 1998. Dissociation of the neural correlates of implicit and explicit memory. Nature 392 (6676), 595-598.

Rugg, M.D., Allan, K., Birch, C.S., 2000. Electrophysiological evidence for the modulation of retrieval orientation by depth of study processing. J. Cogn. Neurosci. 12 (4), 664-678.

Schacter, D.L., Tulving, E., 1994. What are the memory systems of 1994. In: Schacter, D.L., Tulving, E. (Eds.), Memory Systems, pp. 1-39.

Schacter, D.L., 1999. The seven sins of memory-insights from psychology and cognitive neuroscience. Am. Psychol. 54, 182-203.

Simons, J.S., Graham, K.S., Galton, C.J., Patterson, K., Hodges, J.R., 2001. Semantic knowledge and episodic memory for faces in semantic dementia. Neuropsychology 15 (1), 101-114.

Squire, L.R., Knowlton, B., 1995. Memory, hippocampus, and brain systems. In: Gazzaniga, M.S. (Ed.), The Cognitive Neuroscience. MIT Press, Cambridge: MA, pp. 825-837.

Temple, C.M., Richardson, P., 2004. Developmental amnesia: a new pattern of dissociation with intact episodic memory. Neuropsychologia 42 (6), 764-781.

Toth, J.P., 1996. Conceptual automaticity in recognition memory: levels-ofprocessing effects on familiarity. Can. J. Exp. Psychol. [Special Issue: Implicit Memory Research in 1996] 50 (1), 123-138.

Tsivilis, D., Otten, L.J., Rugg, M.D., 2001. Context effects on the neural correlates of recognition memory: an electrophysiological study. Neuron 31 (3), 497-505.

Tulving, E., 1972. Episodic and semantic memory. In: Tulving, E., Donaldson, W. (Eds.), Organisation of Memory. Academic Press, New York, pp. 381-403.

Vargha-Khadem, F., Gadian, D.G., Watkins, K.E., Connelly, A., Van Paesschen, W., Mishkin, M., 1997. Differential effects of early hippocampal pathology on episodic and semantic memory. Science 277 (5324), 376-380.

Warrington, E.K., McCarthy, R., 1983. Category specific access dysphasia. Brain 106 (4), 859-878.

Wilding, E.L., Doyle, M.C., Rugg, M.D., 1995. Recognition memory with and without retrieval of context: an event-related potential study. Neuropsychologia 33 (6), 743-767.

Windmann, S., Urbach, T.P., Kutas, M., 2002. Cognitive and neural mechanisms of decision biases in recognition memory. Cereb. Cortex 12 (8), 808-817.

Wolk, D.A., Schacter, D.L., Berman, A.R., Holcomb, P.J., Daffner, K.R., Budson, A.E., 2004. An electrophysiological investigation of the relationship between conceptual fluency and familiarity. Neurosci. Lett. 369 (2), 150-155.

Yonelinas, A.P., 1997. Recognition memory ROCs for item and associative information: the contribution of recollection and familiarity. Mem. Cogn. 25, 747-763.

Yonelinas, A.P., 2001. Components of episodic memory: the contribution of 
recollection and familiarity. Philos. Trans. R. Soc. Lond., Ser. B Biol. Sci. 356, 1363-1374.

Yonelinas, A.P., 2002. The nature of recollection and familiarity: a review of 30 years of research. J. Mem. Lang. 46 (3), 441-517.

Yonelinas, A.P., Kroll, N.E.A., Dobbins, I.G., Soltani, M., 1999. Recogni- tion memory for faces: when familiarity supports associative recognition judgments. Psychon. Bull. Rev. 6, 654-661.

Yovel, G., Paller, K.A., 2004. The neural basis of the butcher-on-the-bus phenomenon: when a face seems familiar but is not remembered. NeuroImage 21 (2), 789-800. 